\title{
Testing Spatial Autocorrelation in Weighted Networks: the Modes Permutation Test
}

\author{
François Bavaud \\ Department of Computer Science and Mathematical Methods \\ Department of Geography \\ University of Lausanne, Switzerland
}

\begin{abstract}
In a weighted spatial network, as specified by an exchange matrix, the variances of the spatial values are inversely proportional to the size of the regions. Spatial values are no more exchangeable under independence, thus weakening the rationale for ordinary permutation and bootstrap tests of spatial autocorrelation. We propose an alternative permutation test for spatial autocorrelation, based upon exchangeable spatial modes, constructed as linear orthogonal combinations of spatial values. The coefficients obtain as eigenvectors of the standardised exchange matrix appearing in spectral clustering, and generalise to the weighted case the concept of spatial filtering for connectivity matrices. Also, two proposals aimed at transforming an acessibility matrix into a exchange matrix with with a priori fixed margins are presented. Two examples (inter-regional migratory flows and binary adjacency networks) illustrate the formalism, rooted in the theory of spectral decomposition for reversible Markov chains.
\end{abstract}

Keywords: bootstrap, local variance, Markov and semi-Markov processes, Moran's I, permutation test, spatial autocorrelation, spatial filtering, weighted networks

\section{Introduction}

Permutation tests of spatial autoccorelation are justified under exchangeability, that is the premise that the observed scores follow a permutation-invariant joint distribution. Yet, in the frequently encountered case of geographical data collected on regions differing in importance, the variance of a regional score is expected to decrease with the size of the region, in the same way that the variance of an average is inversely proportional to the size of the sample in elementary statistics: heteroscedasticity holds in effect, already under spatial independence, thus weakening the rationale of the celebrated spatial autocorrelation permutation test (e.g. Cliff and Ord 1973; Besag and Diggle 1977) in the case of a weighted network.

This paper presents an alternative permutation test for spatial autocorrelation, whose validity extends to the weighted case. The procedure relies upon spatial modes, that is linear orthogonal combinations of spatial values, originally 
based upon the eigenvectors of the standardized connectivity or adjacency matrix (Tiefelsdorf and Boots 1995; Griffth 2000). In contrast to regional scores, the variance of the spatial modes turn out to be constant under spatial independence, thereby justifying the modes permutation test for spatial autocorrelation.

Section 2.1 presents the definition of the local variance and Moran's I in the arguably most general setup for spatial autocorrelation, based upon the normalised, symmetrical exchange matrix, whose margins define regional weights (Bavaud 2008a). Section 2.2 presents, in the spirit of spatial filtering (Griffth 2000), the spectral decomposition of the exchange matrix, or rather of a standardised version of it, currently used in spectral graph theory (Chung 1997; von Luxburg 2007; Bavaud 2010), supplying the orthogonal components defining in turn the spatial modes. Section 2.3 presents the mode permutation test, and its bootstrap variant, illustrated in section 2.4 on Swiss migratory and linguistic data.

Section 3 addresses the familiar case of binary or weighted adjacency matrices, which have to be first converted into exchange matrices with a priori fixed margins. Two proposals, namely a simple rescaling with diagonal adjustment (Section 3.1) and the construction of time-embeddable exchange matrices (Section 3.2) are presented, and illustrated on the popular "distribution of Blood group A in Eire" dataset (Section 3.3).

\section{Spatial autocorrelation in weighted netwoks}

\subsection{Local covariance and the exchange matrix}

Consider a set of $n$ regions with associated weights $f_{i}>0$, normalized to $\sum_{i=1}^{n} f_{i}=1$. Weights measure the importance of the regions, and define weighted regional averages and variances as

$$
\bar{x}:=\sum_{i=1}^{n} f_{i} x_{i} \quad \operatorname{var}(x):=\sum_{i=1}^{n} f_{i}\left(x_{i}-\bar{x}\right)^{2}=\frac{1}{2} \sum_{i j} f_{i} f_{j}\left(x_{i}-x_{j}\right)^{2} .
$$

Here $x=\left(x_{i}\right)$ represents a density variable or a spatial field, that is a numerical quantity attached to region $i$, transforming under aggregation $i, j \rightarrow[i \cup j]$ as $x_{[i \cup j]}=\left(f_{i} x_{i}+f_{j} x_{j}\right) /\left(f_{i}+f_{j}\right)$, as for instance "cars per inhabitants", "average income" or "proportion of foreigners".

The last identity in (1) is straightforward to check (Lebart 1969), and shows the variance to measure the average squared dissimilarity between pairs $(i, j)$ of regions, selected independently with probability $f_{i} f_{j}$. A more general sampling scheme consists in selecting the regional pair $(i, j)$ with probability $e_{i j}$, such that

$$
e_{i j} \geq 0 \quad e_{i j}=e_{j i} \quad e_{i \bullet}:=\sum_{j} e_{i j}=f_{i} \quad e_{\bullet \bullet}=1
$$

where "•" denotes the summation over the replaced index. A $n \times n$ matrix $E=\left(e_{i j}\right)$ obeying (2) is called an exchange matrix (Berger and Snell 1957; 
Bavaud 2008a), compatible with the regional weights $f$. The exchange matrix defines an undirected weighted network, with edges weights $e_{i j}$ and regional weights $f_{i}=e_{i}$. It contains loops in general $\left(e_{i i} \geq 0\right)$, denoting regional selfinteraction or autarachy (Bavaud 1998).

By construction, the exchange matrix generates a reversible Markov transition matrix $W=\left(w_{i j}\right)$ (Bavaud 1998) with stationary distribution $f$ :

$$
w_{i j}:=\frac{e_{i j}}{f_{i}} \geq 0 \quad \sum_{j} w_{i j}=1 \quad \sum_{i} f_{i} w_{i j}=f_{j} \quad f_{i} w_{i j}=f_{j} w_{j i}=e_{i j} .
$$

$W$ constitutes a row-normalized matrix of spatial weights, entering in the autoregressive models of spatial econometrics (see e.g. Anselin 1988; Cressie 1993; Leenders 2002; Haining 2003; Arbia 2006; LeSage and Pace 2009).

In spatial applications, the components of the exchange matrix are large for nearby regions and small for regions far apart. The quantity

$$
\operatorname{var}_{\mathrm{loc}}(x):=\frac{1}{2} \sum_{i j} e_{i j}\left(x_{i}-x_{j}\right)^{2}
$$

defines the local variance, that is the average squared dissimilarity between neighbours. Comparing the local and the ordinary (weighted) variance defines Geary's $c$ and Moran's I, measuring spatial autocorrelation (e.g. Geary 1954; Moran 1950 ; Cliff and Ord 1973; Tiefelsdorf and Boots 1995; Anselin 1995). Namely, $c(x):=\operatorname{var}_{\text {loc }}(x) / \operatorname{var}(x)$ (differing from its usual variant by a factor $n /(n-1)$ ) and

$$
I(x):=1-c(x)=\frac{\operatorname{var}(x)-\operatorname{var}_{\text {loc }}(x)}{\operatorname{var}(x)}=\frac{\sum_{i j} e_{i j}\left(x_{i}-\bar{x}\right)\left(x_{j}-\bar{x}\right)}{\sum_{i} f_{i}\left(x_{i}-\bar{x}\right)^{2}} .
$$

\subsection{Spatial filtering and spatial modes}

Spatial filtering primarily aims at visualizing and extracting the latent factors involved in spatial autocorrelation (Tiefelsdorf and Boots 1995; Griffith 2000, 2003; Griffith and Peres-Neto 2006; Chun 2008; Dray 2011; and references therein).

Its first step consists in spectrally decomposing a matrix expressing interregional connectivity in some way or another, such as the adjacency matrix or the exchange matrix. Various choices are often equivalent under uniform weighting of the regions, but the general weighted case calls for more precision. Arguably, the most fruitful decomposition considers the so-called standardized exchange matrix $E^{s}$, with components (Chung 1997, von Luxburg 2007; Bavaud 2010)

$$
e_{i j}^{s}=\frac{e_{i j}-f_{i} f_{j}}{\sqrt{f_{i} f_{j}}} \quad \text { i.e. } \quad E^{s}=\Pi^{-\frac{1}{2}}\left(E-f f^{\prime}\right) \Pi^{-\frac{1}{2}} \quad \text { with } \quad \Pi=\operatorname{diag}(f) .
$$

Its spectral decomposition

$$
E^{s}=U \Lambda U^{\prime} \quad \text { with } \quad U=\left(u_{i \alpha}\right) \text { orthogonal and } \Lambda=\operatorname{diag}(\lambda) \text { diagonal }
$$


generates a trivial eigenvalue $\lambda_{0}=0$ associated with the trivial eigenvector $u_{i 0}=\sqrt{f_{i}}$. The remaining non-trivial decreasingly ordered eigenvalues $\lambda_{\alpha}$ (for $\alpha=1, \ldots, n-1)$ lie in the interval $[-1,1]$, as a consequence of the PerronFroebenius theorem and the symmetry of $E^{s}$.

Also, $\lambda_{1}=1$ iff $E$ is reducible, that is consisting of two or more disconnected components (Figure 1), and $\lambda_{n-1}=-1$ iff $E$ is bipartite, i.e. partitionable into two sets without within connections (e.g. Kijima 1997; Aldous and Hill 2002).
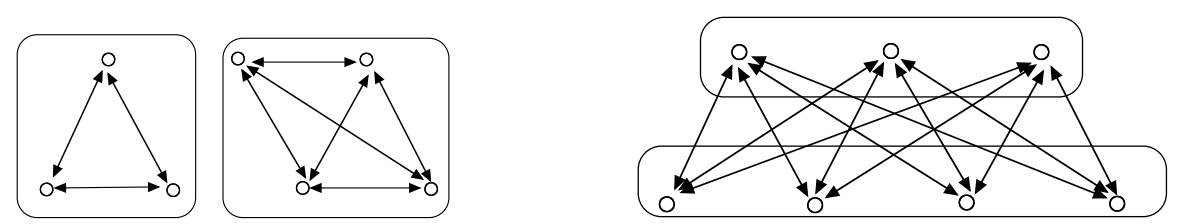

Fig. 1. Reducible network, with $\lambda_{1}=1$ (left) and bipartite network, with $\lambda_{n-1}=-1$ (right)

The exchange matrix itself expresses as

$$
e_{i j}=f_{i} f_{j}+\sqrt{f_{i} f_{j}} \sum_{\alpha=1}^{n-1} \lambda_{\alpha} u_{i \alpha} u_{j \alpha}=f_{i} f_{j}\left[1+\sum_{\alpha \geq 1} \lambda_{\alpha} c_{i \alpha} c_{j \alpha}\right]
$$

where the raw coordinates

$$
c_{i \alpha}:=\frac{u_{i \alpha}}{u_{i 0}}=\frac{u_{i \alpha}}{ \pm \sqrt{f_{i}}}
$$

can be used at visualizing distinct levels of spatial autocorrelation (Griffith 2003), or at specifying the positions of the $n$ regions in a factor space (Bavaud 2010).

Raw coordinates are orthogonal and standardized, in the sense

$$
\sum_{i} f_{i} c_{i \alpha}=\delta_{\alpha 0} \quad \sum_{i} f_{i} c_{i \alpha} c_{i \beta}=\delta_{\alpha \beta} .
$$

As a consequence, the $n$ regional values $x$ can be converted into $n$ modal values $\hat{x}$, and vice-versa, as

$$
\hat{x}_{\alpha}:=\sum_{i} f_{i} c_{i \alpha} x_{i} \quad x_{i}=\sum_{\alpha \geq 0} c_{i \alpha} \hat{x}_{\alpha}=\bar{x}+\sum_{\alpha \geq 1} c_{i \alpha} \hat{x}_{\alpha} .
$$

Equations (8) express orthogonal, Fourier-like correspondence between regional values and modes. The latter depict global patterns, integrating the contributions from all regions. In particular, the trivial mode yields the field average: $\hat{x}_{0}=\bar{x}$.

Borrowing an analogy from solid-state Physics, the spatial field $x$ can describe the individual displacements of each of the $n$ atoms of a crystal. The modes $\hat{x}$ 
then provide global parameters describing the collective motion of atoms, consisting of a superposition of sound waves or harmonics, whose specific eigenfrequencies are determined by the nature of the crystal, and whose knowledge permit to reconstruct the individual atomic displacements.

\subsection{The modes permutation test}

2.3.1 Heterodasticity of the spatial field The hypothesis $H_{0}$ of spatial independence requires the covariance matrix of the spatial field $X=\left(X_{1}, \ldots, X_{n}\right)$ to be diagonal with components inversely proportional to the spatial weights, that is of the form (see the Appendix)

$$
\sigma_{i j}=\operatorname{Cov}\left(X_{i}, X_{j}\right)=\delta_{i j} \frac{\sigma^{2}}{f_{i}} \quad \text { where } \quad \sigma^{2}=\operatorname{Var}(\bar{X})
$$

In its usual form, the direct or regional permutation test compares the observed value of Moran's $I(x)$ to a set of values $I(\pi(x))$, where $\pi(x)$ denotes a permutation (that is a sampling without replacement) of the $n$ regional values $x$ (e.g. Cliff and Ord 1973; Thioulouse et al. 1995; Li and al. 2007; Bivand et al. 2009a). Sampling with replacement, generating bootstrap resamples can also be carried out.

Both procedures are justified by the fact that the spatial variables $X_{i}$ are identically distributed under $H_{0}$. Yet, (9) shows the latter assertion to be wrong whenever the regional weights differ, thus jeopardizing the rationale of the direct approach, based upon the permutation or the bootstrap of regional values.

The possible heteroscedasticity of regional values has been addressed by quite a few researchers, in particular in epidemiology, and various proposals (transformations of variables or weights, reformulations in terms of residuals, Bayesian approaches) have been investigated (see e.g. Waldhör 1996, Assunção and Reis 1999 or Haining 2003).

2.3.2 Homoscedasticity of the spatial modes As annouced in the introduction, this paper proposes a presumably new modal test, identical in spirit to the direct test but based upon modes permutation, together with a variant based upon modes bootstrap. Its existence results from two fortunate circumstances (see the Appendix), namely i) the homoscedasticity of the modes under $H_{0}$

$$
\hat{\sigma}_{\alpha \beta}:=\operatorname{Cov}\left(\hat{X}_{\alpha}, \hat{X}_{\beta}\right)=\delta_{\alpha \beta} \sigma^{2} \quad \text { with } \quad \hat{X}_{\alpha}=\sum_{i} f_{i} c_{i \alpha} X_{i}
$$

and ii) the simplicity of Moran's $I$ expression in terms of spatial modes, which reads

$$
I(x) \equiv I(\hat{x})=\frac{\sum_{\alpha \geq 1} \lambda_{\alpha} \hat{x}_{\alpha}^{2}}{\sum_{\alpha \geq 1} \hat{x}_{\alpha}^{2}} .
$$

As expected, the trivial mode $\hat{x}_{0}=\bar{x}$ does not contribute to Moran's $I$. Under $H_{0}$, its expectation and variance under all remaining $(n-1)$ ! non-trivial modes 
permutations read (see the Appendix)

$$
\begin{gathered}
E_{\pi}(I \mid \hat{x})=\frac{1}{n-1} \sum_{\alpha \geq 1} \lambda_{\alpha}=\frac{\operatorname{trace}(W)-1}{n-1} \geq \frac{-1}{n-1} \\
\operatorname{Var}_{\pi}(I \mid \hat{x})=\frac{s(\hat{x})-1}{(n-1)(n-2)}\left[\sum_{\alpha \geq 1} \lambda_{\alpha}^{2}-\frac{1}{n-1}\left(\sum_{\alpha \geq 1} \lambda_{\alpha}\right)^{2}\right]
\end{gathered}
$$

where

$$
s(\hat{x}):=(n-1) \frac{\sum_{\alpha \geq 1} \hat{x}_{\alpha}^{4}}{\left(\sum_{\alpha \geq 1} \hat{x}_{\alpha}^{2}\right)^{2}} \geq 1
$$

is a measure of modes dispersion.

2.3.3 The test The modes autocorrelation test consists in refuting $H_{0}$, which denies spatial dependence, if the value (11) of $I(\hat{x})$ is extreme w.r.t. the sample $\{I(\pi(\hat{x}))\}$ of $B$ permuted or boostrapped mode values, that is if its quantile is near 1 (evidence of positive autocorrelation) or near 0 (negative autocorrelation).

As expected, the trivial mode $\hat{x}_{0}=\bar{x}$ does not contribute to Moran's $I$. Also, $I(x)$ together with its permuted or boostrapped values lie in an interval comprised in $\left[\lambda_{n-1}, \lambda_{1}\right] \subseteq[-1,1]$. The interval reduces to a single point $I(\pi(\hat{x})) \equiv$ $I_{0}$, invariant under permutations or boostraping, with a corresponding variance (13) of zero, thus ruining the autocorrelation test, if (see 11, 13):

a) $s(\hat{x})=1$, that is $\hat{x}_{\alpha}^{2} \equiv \hat{x}^{2}$ or equivalently $\hat{x}_{\alpha} \equiv \epsilon_{\alpha} \hat{x}$, where $\hat{x} \in \mathbb{R}$ and $\epsilon_{\alpha}= \pm 1$ for all $\alpha \geq 1$. Following (8), this occurs for "untestable" spatial fields of the form $x_{i}=\bar{x}+\hat{x} z_{i}$ where $z_{i}=\sum_{\alpha \geq 1} \epsilon_{\alpha} u_{i \alpha} / u_{i 0}$ actually defines a set of $2^{n-1}$ configurations depending on the choice of the $\epsilon_{\alpha}$, whose sign is arbitrary, as is the sign of the eigenvectors $u_{\alpha}$.

Noticeably, the constant field $x_{i} \equiv \bar{x}$ is untestable, with a value $I(x)=$ $0 / 0$ not even defined. However intriguing, the empirical relevance of those "untestable" spatial fields is debatable, in view of the vanishing probability to encounter exactly such a spatial pattern.

b) $\lambda_{\alpha} \equiv \lambda$ for all $\alpha \geq 1$, as with the :

i) frozen networks $E:=E^{(0)}$, where $e_{i j}^{(0)}:=f_{i} \delta_{i j}$ is the disconnected graph $^{1}$, associated to the immobile Markov chain with $\lambda_{\alpha} \equiv 1$ and $I(x) \equiv 1$ (Figure 2 left)

ii) or as with the perfectly mobile networks $E:=E^{(\infty)}$, where $e_{i j}^{(\infty)}:=$ $f_{i} f_{j}$ is the complete weighted graph, free of distance-deterrence effects, associated to the memoryless Markov chain with $\lambda_{\alpha} \equiv 0$ and $I(x) \equiv 0$ (Figure 2 middle)

\footnotetext{
${ }^{1}$ Here the notations match the higher-order discrete time extensions of the exchange matrix, resulting (under weak regularity conditions) from the iteration of the Markov transition matrix as

$$
E^{(r)}:=\Pi W^{r} \quad E^{(0)}=\Pi \quad E^{(2)}=E \Pi^{-1} E \quad E^{(\infty)}=f f^{\prime} .
$$
}


as well as with their linear combinations $E:=a E^{(0)}+(1-a) E^{(\infty)}$. Also, networks made of $n=2$ regions are untestable: they automatically satisfy b) and a) above (Figure 2 right).

Under the additional normal assumption $\hat{X}_{\alpha} \sim N\left(0, \sigma^{2}\right)$ for $\alpha \geq 1$, one can show $E(s(\hat{x}))=3(n-1) /(n+1)$ by the Pitman-Koopmans theorem (Cliff and Ord 1981 p.43) and hence

$$
\begin{aligned}
E\left(\operatorname{Var}_{\pi}(I \mid \hat{x})\right) & =\frac{2}{n^{2}-1}\left[\sum_{\alpha \geq 1} \lambda_{\alpha}^{2}-\frac{1}{n-1}\left(\sum_{\alpha \geq 1} \lambda_{\alpha}\right)^{2}\right] \\
& =\frac{2}{n^{2}-1}\left[\operatorname{tr}\left(W^{2}\right)-1-\frac{(\operatorname{tr}(W)-1)^{2}}{n-1}\right] .
\end{aligned}
$$

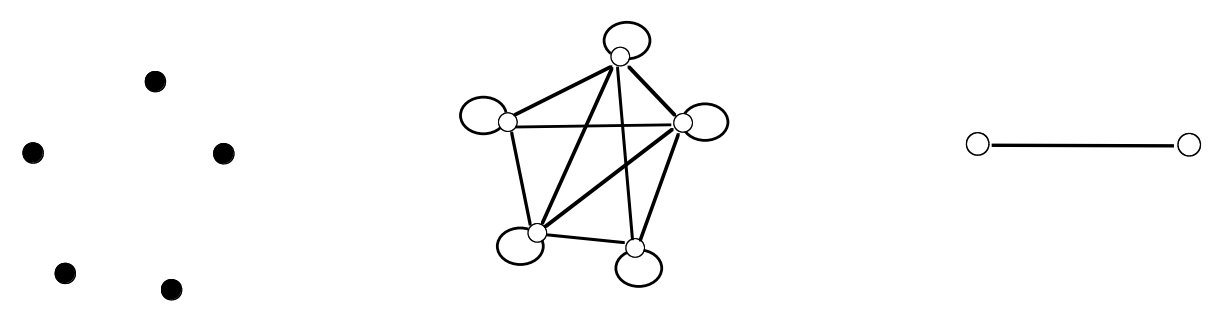

Fig. 2. Moran's $I(x)$ is constant, independent of the value of the field $x$ for the disconnected or frozen network (left), for the fully connected or perfectly mobile network (middle), and their linear combinations. Its minimum $I(x) \equiv-1$ occurs for the loopless network with $n=2$ (right).

\subsection{Illustration: Swiss migratory and linguistic data}

Flows constitute a major source of exchange matrices (e.g. Goodchild and Smith 1980; Willekens J. 1983; Fotheringham and O'Kelly, M.E. 1989; Sen and Smith 1995; Bavaud 1998, 2002). Let $n_{i j}(T)$ denote the number of units (people, goods, matter, etc.) initially in region $i$ and located in region $j$ after a time T. Quasisymmetric flows are of the form $n_{i j}=a_{i} b_{j} c_{i j}$ with $c_{i j}=c_{j i}$, as predicted by Gravity modelling. They generate reversible spatial weights $w_{i j}:=n_{i j} / n_{i \bullet}$, with stationary distribution $f_{i}$, whose product $f_{i} w_{i j}$ defines the exchange matrix $e_{i j}$ (Bavaud 2002).

Consider the inter-regional migrations data $n_{i j}(T)$ between the $n=26$ Swiss cantons for $T=5$ years (1985-90), together with the spatial fields $x=$ "proportion of germanophones" or $x=$ "proportion of anglophones", for each canton. After determining the quasi-symmetric ML estimates $\hat{n}_{i j}$ (Bavaud 2002), the exchange matrix is computed, and so are the spatial modes $\hat{x}_{\alpha}$ from (8) and Moran's 
index $I(\hat{x})$ from (11). Figure 3 depicts the distribution of $10^{\prime} 000$ permutation and boostrap resamples of $I(\pi(\hat{x}))$, from which the bilateral $p$-values of Table 1 can be computed (see Section 3.2 for the details).
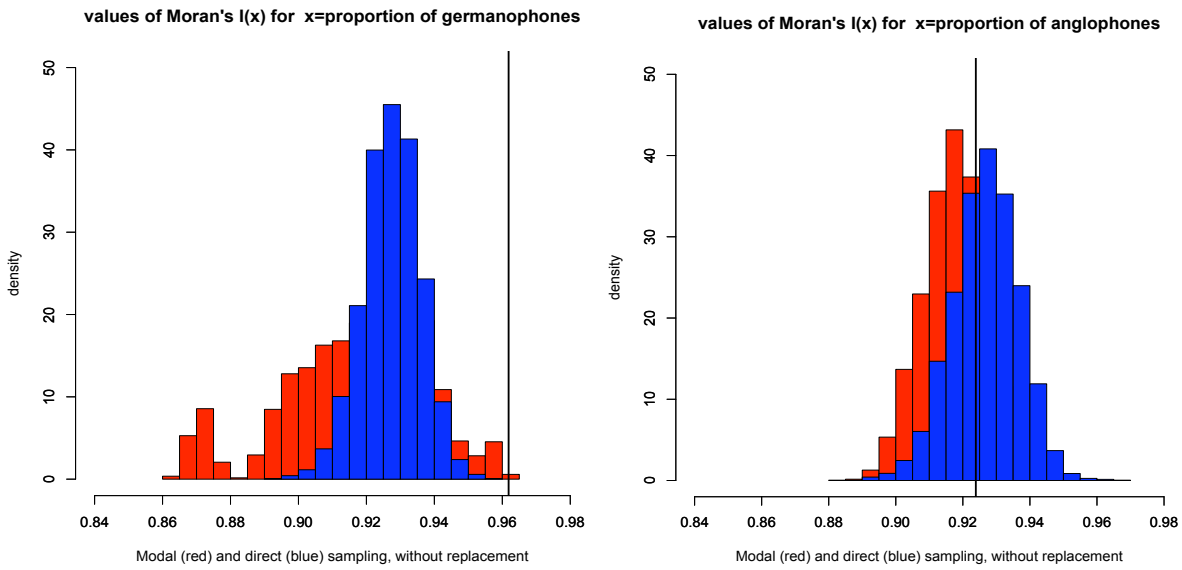

values of Moran's $I(x)$ for $x=$ proportion of germanophones
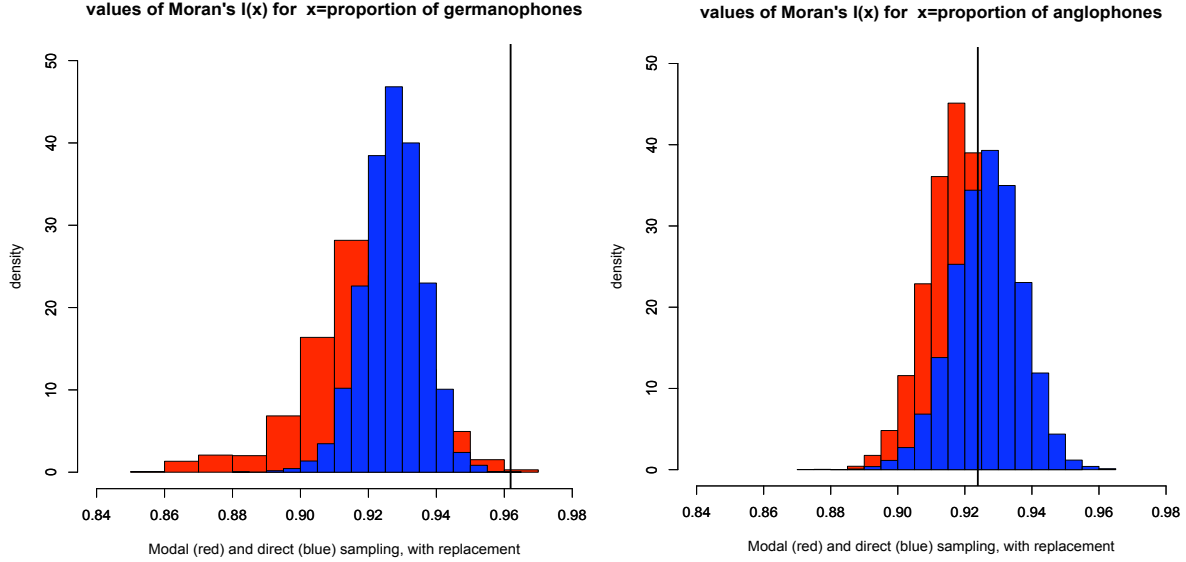

Fig. 3. Permutation (above) and bootstrap (below), modal (red) and regional (blue) testing of the "migation-driven" spatial autocorrelation among germanophones (left) and anglophones (right). $B=10^{\prime} 000$ samples are generated each time, and compared with the observed $I$, marked vertically.

Most people do not migrate towards other cantons in five years, thus making the exchange matrix "cold" (that is close to the frozen $E^{(0)}$ ), with a dominating diagonal, accounting for the high values of $I$ and $E_{\pi}(I)$ in Table 1.

Swiss native linguistic regions divide into German, French and Italian. Migrants tend to avoid to cross the linguistic barriers, thus accounting for the 


\begin{tabular}{|r|l|l|l|l|l|}
\hline & germanophones & anglophones \\
\cline { 3 - 5 }$E_{\pi}(I)$ & 0.962 & 0.917 & 0.924 & 0.917 \\
\cline { 3 - 5 }$E_{\pi}(I \mid \hat{x})$ & 0.00044 & 0.000082 & modal permutation & .0006 & .048 \\
$z$ & 2.13 & 0.74 & regional permutation & .0002 & .075 \\
$\operatorname{Var}_{(\hat{x})}$ & 11.29 & 2.90 & modal bootstrap & .0036 & .46 \\
regional bootstrap & .0004 & .76 \\
\hline
\end{tabular}

Table 1. Left: observed $I$, its modal permutation expectation (12) and variance (13) together with the $z$-value $z:=\left(I-E_{\pi}(I)\right) / \sqrt{\operatorname{Var}_{\pi}(I \mid \hat{x})}$ and $s(\hat{x})$ in (13). Right: $p$ values associated to the modal and direct autocorrelation test, in their permutation and bootstrap variants.

spatial autocorrelation of "germanophones" (Table 1, right). Detecting spatial patterns in the anglophones repartition is, as expected from the above migratory scheme, less evident.

Bootstrap tests (modal or regional) appear here less powerful than permutation tests - a possibly true conjecture in general (Corcoran and Mehta 2002; Janssen and Pauls 2005).

Modal autocorrelation tests of "anglophones" seem more sensitive than their regional conterparts, while the opposite holds for "gemanophones": the usual test of autocorrelation underestimates the dispersion of the resampled values of $I(\pi$ (germanophones) $)$ (Figure 3, left), thus inflating the risk of type I errors for size-unadjusted Moran's I, in accordance with the simulation results of Assunção and Reis (1999).

\section{$3 \quad$ Adjacency graphs and accessibilities}

Very commonly, space is defined by a binary, off-diagonal and symmetric connectivity or adjacency matrix $n \times n$ matrix $A=\left(a_{i j}\right)$, specifying whether distinct regions $i$ and $j$ are direct neighbours $\left(a_{i j}=1\right)$ or not $\left(a_{i j}=0\right)$. This scheme can also, as in gravity modelling, be extended to "weighted adjacencies" or $a c$ cessibilities $a_{i j}=f\left(d_{i j}\right)$ defined by a non-negative distance deterrence function $f\left(d_{i j}\right)$ decreasing with the distance $d_{i j}$ between distinct regions $i$ and $j$.

In the sequel, we consider accessibility matrices with $a_{i j} \geq 0, a_{i j}=a_{j i}$ and $a_{i i}=0$, with the interpretation that distinct regions $i$ and $j$ are direct neighbours iff $a_{i j}>0$. By construction, the three quantities

$$
\varepsilon_{i j}=\frac{a_{i j}}{a_{\bullet \bullet}} \quad \kappa_{i j}=\frac{a_{i j}}{a_{i \bullet}} \quad \sigma_{i}=\frac{a_{i \bullet}}{a_{\bullet \bullet}}=\varepsilon_{i \bullet}
$$

respectively constitute an exchange matrix, its associated transition matrix and the stationary distribution, proportional to the (possibly weighted) number of neighbours or degree.

Although the series of steps of Section 2 can be wholly carried out by adopting $\varepsilon:=\left(\varepsilon_{i j}\right)$ as the reference exchange matrix, this procedure reveals itself far form satisfactory in general: exchanges between non-adjacent regions are precluded, 
as are the diagonal exchanges, thus mechanically generating negative eigenvalues in view of $0=\operatorname{trace}\left(\varepsilon^{s}\right)=1+\sum_{\alpha>1} \lambda_{\alpha}$. Even worse, the normalized degree $\sigma$ in (15), reflecting the regions centrality, strongly differs in general from the regions weights $f$, reflecting their importance: a densely populated region can be weakly connected to the rest of the territory, and inversely.

Proposals $\mathrm{A}$ and $\mathrm{B}$ below aim at converting an accessibility matrix $A$ into an exchange matrix $E$ with given margins $f$, while keeping the neighborhood structure expressed by $A$ as intact as possible.

\subsection{Proposal A: simple rescaling with diagonal adjustment}

Define the symmetric exchange matrix

$$
e_{i j}:= \begin{cases}C b_{i} b_{j} a_{i j} & \text { if } i \neq j \\ h_{i} & \text { otherwise. }\end{cases}
$$

where $C, b$ and $h$ are non-negative quantities obeying the normalisation condition (recall $\left.a_{i i}=0\right)$

$$
C b_{i} \sum_{j} a_{i j} b_{j}+h_{i}=f_{i} \quad \text { for all } i .
$$

By construction, $e_{i \bullet}=f_{i}$ and, for $i \neq j, e_{i j}=0$ whenever $a_{i j}=0$.

An obvious choice, among many possibilities, consists in defining $b$ as the first normalised eigenvector of the accessibility matrix $A$, that is obeying $A b=\mu b$, where $\mu>0$ is the largest eigenvalue of $A$, and $b$ (normalised to $\sum_{i} b_{i}^{2}=1$ ) is non-negative by the Perron-Froebenius theorem on non-negative matrices. $b_{i}$ ( or $b_{i}^{2}$ ) is a measure of relative centrality of region $i$, sometimes referred to as eigenvector centrality in the social networks literature.

Condition (17) becomes $C \mu b_{i}^{2}+h_{i}=f_{i}$, implying $C=(1-\eta) / \mu$, where the quantity $\eta:=\sum_{i} h_{i}$ fixes the diagonal parameters to $h_{i}=f_{i}-(1-\eta) b_{i}^{2}$, and ranges in $\eta \in[H, 1]$ to insure the non-negativity of $C$ and $h$, where $H:=$ $1-\min _{i}\left(f_{i} / b_{i}^{2}\right) \geq 0$.

The free parameter $\eta$ controls the "autarchy of the network": in the limit $\eta \rightarrow 1$, one recovers the frozen network of Section 2.3.3, while $\eta \rightarrow H$ yields at least one region with $e_{i i}=0$. Note that $e_{i i}=0$ cannot hold for all regions, unless $b \equiv \sqrt{f}$ precisely, in which case $H=0$.

\subsection{Proposal B: time-embeddable exchange matrices}

The second proposal is based upon the observation that $\kappa_{i j}$ in (15) constitutes a jump transition matrix, defining the probability that $j$ will be the next, distinct region to be visited after having been in region $i$ (recall $\kappa_{i i}=0$ ). Suppose in addition that, once arrived in $j$, the state remains in $j$ for a certain random time $t_{j}$ with cumulative distribution function $F_{j}(t)$, with average waiting time or sojurn time $\tau_{j}=\int t d F_{j}(t)$. This set-up precisely defines a so-called semiMarkov process (e.g. Çinlar 1975, Barbu and Limnios 2008). 
Together, the stationary distribution $\sigma_{j}(15)$ of the jump transition matrix $\kappa_{i j}$ and the sojurn times $\tau_{j}$ determine the fraction of time spend in region $j$, that is the regional weight $f_{j}$, as (see e.g. Bavaud 2008b)

$$
f_{j}=\frac{\sigma_{j} \tau_{j}}{\tau} \quad \text { where } \quad \tau:=\sum_{j} \sigma_{j} \tau_{j} \quad \text { or equivalently } \quad \frac{1}{\tau}=\sum_{j} \frac{f_{j}}{\tau_{j}} .
$$

Furthermore, requiring exponentially distributed random times $t_{j}$ ensures the semi-Markov process to be continuous or time-embeddable, that is of the form $W(t)=\exp (t R)$ (matrix exponential) where $R=\left(r_{i j}\right)$ is the $n \times n$ rate transition matrix, with components $r_{i j}=\left(\kappa_{i j}-\delta_{i j}\right) / \tau_{i}$. In particular,

$$
\sum_{j} r_{i j}=0 \quad \quad \sum_{i} f_{i} r_{i j}=0 .
$$

The existence of transition matrices $W(t)=\left(w_{i j}(t)\right)$ defined for any continuous time $t \geq 0$, rather than limited to integer values $t=0,1,2, \ldots$, characterises timeembeddable Markov chains. The symmetry of the associated exchange matrices $e_{i j}(t):=f_{i} w_{i j}(t)$ follows from the reversibility of $W(t)$, itself insured by the reversibility of the jump matrix.

In summary, proposal B considers the adjacency matrix $A$ as an infinitesimal generator of the exchange matrix $E$; tuning the freely adjustable sojurn times $\tau_{j}$ in (18) permits to transform any degree distribution $\sigma$ into any given regional weights $f$, as required.

To achieve the pratical, numerical construction of the time-embeddable exchange matrix $E(t)$, consider the "standardised rate matrix" $Q=\left(q_{i j}\right)$ with components

$$
q_{i j}:=f_{i}^{\frac{1}{2}} f_{j}^{-\frac{1}{2}} r_{i j}=\frac{\varepsilon_{i j}-\delta_{i j} \sigma_{j}}{\tau \sqrt{f_{i} f_{j}}}
$$

$Q$ is semi-negative definite (see the Appendix). Its eigenvalues $\mu_{\alpha}$ and associated normalised eigenvectors $u_{i \alpha}$ satisfy $\mu_{0}=0$ with $u_{i 0}=\sqrt{f_{i}}$, together with $\mu_{\alpha} \leq 0$ for the non-trivial eigenvalues $\alpha=1, \ldots, n-1$. Now the eigenvectors of the standardized exchange matrix $E^{s}(t)=\left(e_{i j}^{s}(t)\right)(5)$ turn out to be identical to those of $Q$, irrespectively of value of $t$, (see the Appendix), while the non-trivial eigenvalues of $E^{s}(t)$ are related to those of $Q$ by $\lambda_{\alpha}(t)=\exp \left(\mu_{\alpha} t\right)$ for $\alpha=$ $1, \ldots, n-1$. Substituting back in (6) finally yields the exchange matrix as

$$
e_{i j}(t)=f_{i} f_{j}\left[1+\sum_{\alpha \geq 1} \lambda_{\alpha}(t) c_{i \alpha} c_{j \alpha}\right] \quad \text { where } c_{i \alpha}=\frac{u_{i \alpha}}{\sqrt{f_{i}}} \text { and } \lambda_{\alpha}(t)=\exp \left(\mu_{\alpha} t\right)
$$

The eigenvalues $\lambda_{\alpha}(t)$ of the standardized exchange matrix $E^{s}(t)(5)$ are nonnegative. This characterizes continuous-time Markov chain and diffusive processes, by contrast to oscillatory processes associated to negative eigenvalues, as in the bipartite network of Figure 1, or as in the direct accessibility-based approach (15).

As a matter of fact, temporal dependence enters through the quantity $t / \tau$ only: defining $Q^{*}$ as (20) with $\tau=1$ and $\mu^{*}$ as the corresponding non-trivial 
eigenvalues, one gets $\lambda_{\alpha}(t)=\left(\exp \left(\mu_{\alpha}^{*}\right)\right)^{\frac{t}{\tau}}$, which can be directly susbstituted into (11) to compute Moran's $I$. Note the modes $\hat{x}_{\alpha}=\sum_{i} \sqrt{f_{i}} u_{i \alpha} x_{i}$, where the $u_{i \alpha}$ are the eigenvectors of $Q^{*}$, to be time-independant.

The free parameter $t$ (or $t / \tau$ ) represents the (relative) "age of the network". $E(t)$ tends to the frozen network for $t \rightarrow 0$, and to the perfectly mobile network for $t \rightarrow \infty$ (Section 2.3.3). One expects spatial autocorrelation to be more easily detected for small values of $t$, that is for networks able to sustain strong contrasts between local and global variances.
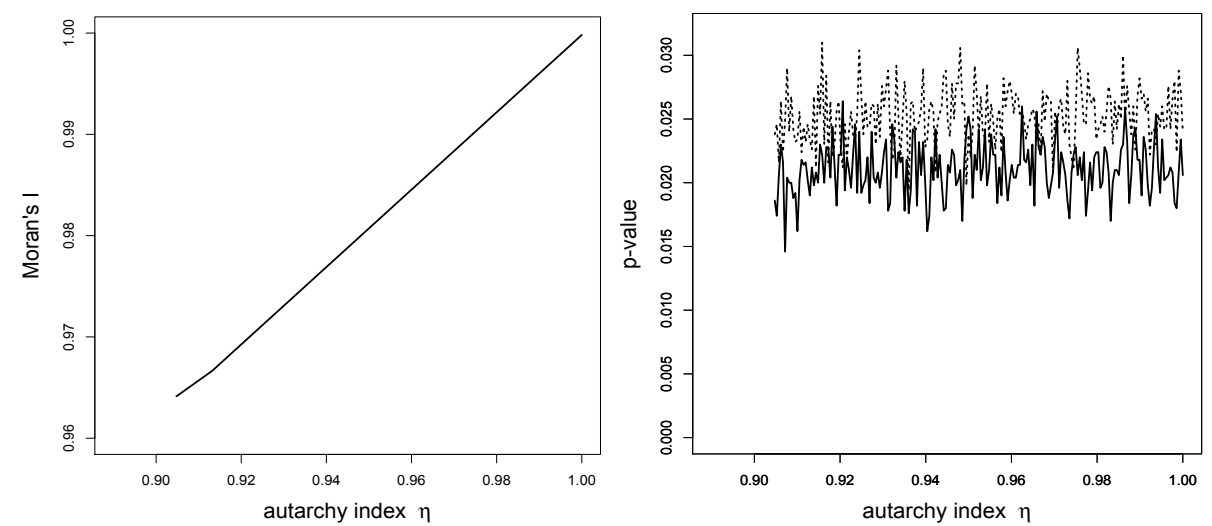

Fig. 4. "Blood group A in Eire" dataset: proposal A. Left: Moran's $I$ as a function of $\eta \in[H, 1]$. Right: two-tailed $p$-values of the modes autocorrelation test, based upon $B=10^{\prime} 000$ permutation (bold line) or bootstrap (dashed line) resamples.

\subsection{Illustration: the distribution of Blood group A in Eire}

Let us revisit the popular "distribution of Blood group A in Eire" dataset (Cliff and Ord 1973; Upton and Fingleton 1985; Griffith 2003; Tiefelsdorf and Griffith 2007), recording the percentage $x$ of the 1958 adult population with of Blood group A in each of the $n=26$ Eire counties, as well as the relative population size $f$, and the inter-regional adjacency matrix $A$ (data from the $\mathrm{R}$ package spdep (Bivand 2009b)).

Following the "proposal A" procedure of Section 3.1 yields $\mu=5.11$ and $H=0.904$, echoing the existence of a region whose weight $f_{i}$ is about ten times smaller than its "eigenvector centrality" $b_{i}^{2}$. Both permutation and boostrap modes autocorrelation tests reveal statistically significant spatial autocorrelation, without obvious dependence upon the autarchy index $\eta$.

"Proposal B" procedure of Section 3.2 yields p-values depicted in Figure 5 , left. As expected, they reveal statistically significant spatial autocorrelation for small values of $t / \tau$, and increase with $t / \tau$. Starting the procedure with one 

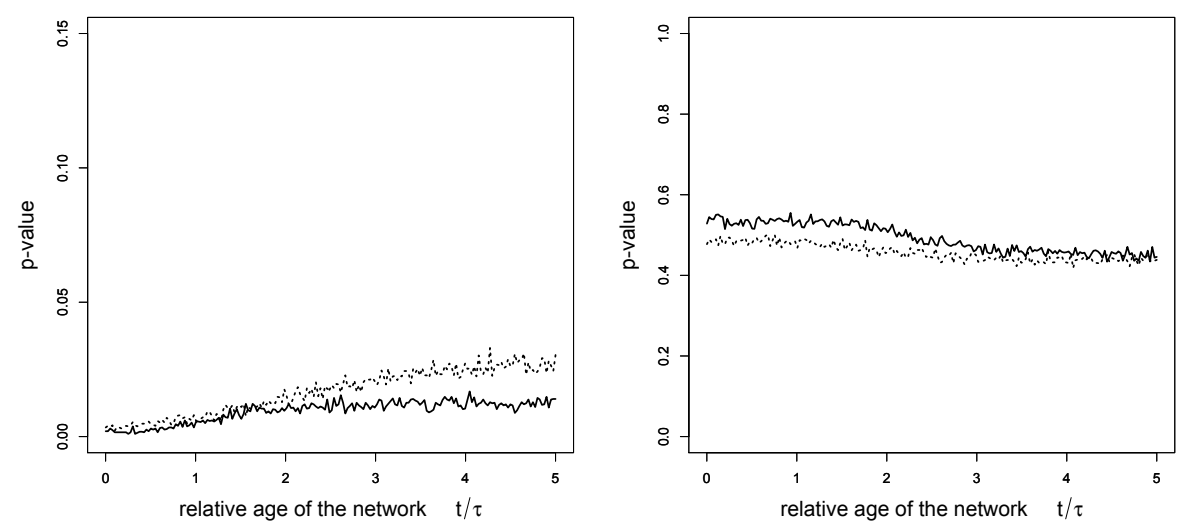

Fig. 5. "Blood group A in Eire" dataset: proposal B. Left: two-tailed $p$-values of the modes autocorrelation test, based upon $B=10^{\prime} 000$ permutation (bold line) or bootstrap (dashed line) resamples. Right: the same procedure, applied to an arbitrarily selected permutation $\pi(x)$ of the original spatial field $x$.

among the many possible permutations of the field produces $p$-values as in Figure 5 (right) and indicates no spatial autocorrelation, as it must.

\section{Conclusion}

Real spatial networks are irregular and subject to aggregation. They are bound to exhibit regions differing in sizes or weights. This paper proposes a weighted analysis of Moran's $I$, in the possibly most general set-up provided by the exchange matrix formalism, rooted in the theory of reversible Markov chains and gravity flows of geographers.

Besides providing a rationale for overcoming the heteroscedasticity problem in the direct application of permutation or boostrap autocorrelation tests, the concept of spatial modes we have elaborated upon arguably generalises the concept of spectrally-based spatial filtering (e.g. Griffith 2003) to a weighted setting, and helps integrating other network-related issues in a unified setting: typically, the first non-trivial raw coordinate $c_{1}$ of Section 2.2 has beeen known for some time to provide the optimal solution to the spectral clustering problem, partitioning a weighted graph into two balanced components (e.g. Chung 1997; von Luxburg 2007; Bavaud 2010).

Finally, local variance (4) can be generalised to local inertias $\frac{1}{2} \sum_{i j} e_{i j} D_{i j}$ (where $D$ represents a squared Euclidean distance between regions) and to local covariances $\frac{1}{2} \sum_{i j} e_{i j}\left(x_{i}-x_{j}\right)\left(y_{i}-y_{j}\right)$, whose future study may hopefully enrich formal issues and applications in spatial autocorrelation. 


\section{References}

Aldous, D. and Fill, J. (2002) Reversible Markov Chains and Random Walks on Graphs. Draft chapters, online version available at http://www.stat.berkeley.edu/users/aldous/RWG/book.html

Anselin, L. (1988) Spatial Econometrics: Methods and Models. Kluwer

Anselin, L. (1995) Local indicators of spatial association - LISA. Geographical Analysis, 27, 93-115

Arbia, G. (2006) Spatial Econometrics: Statistical Foundations and Applications to Regional Convergence. Springer

Assunção and Reis (1999) A new proposal to adjust Moran's I for population density. Statistics in Medicine 18, 2147-2162

Barbu, V.S. and Limnios, N. (2008) Semi-Markov Chains and Hidden Semi-Markov Models toward Applications: Their Use in Reliability and DNA Analysis. Springer

Bavaud, F. (1998) Models for Spatial Weights: a Systematic Look. Geographical Analysis $30,153-171$

Bavaud, F. (2002) The Quasisymmetric Side of Gravity modelling. Environment and Planning A 34, 61-79

Bavaud, F. (2008a) Local concentrations. Papers in Regional Science 87, 357-370

Bavaud, F. (2008b) The Endogenous Analysis of Flows, with Applications to Migrations, Social Mobility and Opinion Shifts. Journal of Mathematical Sociology 32 pp. $239-266$

Bavaud, F. (2010) Multiple Soft Clustering, Spectral Clustering and Distances on Weighted Graphs. Proceedings of the ECML PKDD'10. Lecture Notes in Computer Science 6321, 103-118. Springer.

Berger, J., Snell, J.L. (1957) On the concept of equal exchange. Behavioral Science 2, $111-118$

Besag J., Diggle, P.J. (1977) Simple Monte Carlo Tests for Spatial Pattern. Journal of the Royal Statistical Society (Series C, Applied Statistics) 26, 327-333

Bivand, R. S., Müller, W., Reder, M. (2009a) Power Calculations for Global and Local Moran's I. Computational Statistics and Data Analysis 53, 2859-2872

Bivand, R. (2009b) Applying Measures of Spatial Autocorrelation: Computation and Simulation. Geographical Analysis 41, 375-384

Chun, Y. (2008) Modeling network autocorrelation within migration ßows by eigenvector spatial filtering. J Geogr Syst 10, 317-344

Chung, F.R.K. (1997) Spectral graph theory. CBMS Regional Conference Series in Mathematics 92. American Mathematical Society, Washington

Çinlar, E. (1975) Introduction to Stochastic Processes. Prentice Hall, New York

Cliff, A.D., Ord, J.K., (1973) Spatial autocorrelation. Pion

Cliff, A.D., Ord, J.K., (1981) Spatial Processes: Models \& Applications.

Corcoran, C.D., Mehta, C.R. (2002) Exact level and power of permutation, bootstrap, and asymptotic tests of trend. Journal of Modern Applied Statistical Methods 1, $42-51$

Cressie, N.A.C. (1993) Statistics for Spatial Data. Wiley

Dray, S. (2011) A New Perspective about Moran's Coefficient: Spatial Autocorrelation as a Linear Regression Problem. Geographical Analysis 43, 127-141

Fotheringham, A.S., O'Kelly, M.E. (1989) Spatial Interaction Models: Formulations and Applications. Kluwer

Geary, R. (1954) The Contiguity Ratio and Statistical Mapping, The Incorporated Statistician 5, 115-145 
Goodchild, M.F., Smith, T.R. (1980) Intransitivity, the spatial interaction model, and US migration streams. Environment and Planning A 12, 1131-1144

Griffith, D.A. (2000) Eigenfunction properties and approximations of selected incidence matrices employed in spatial analyses. Linear Algebra and its Applications, Vol.321, pp. $95-112$

Griffith, D.A. (2003) Spatial Autocorrelation and Spatial Filtering: Gaining Understanding through Theory and Scientific Visualization. Springer

Griffith, D.A., Peres-Neto, P.R. (2006) Spatial modeling in ecology: the flexibility of eigenfunction spatial analyses. Ecology 87, 2603-2613

Haining, R. P. (2003) Spatial Data Analysis: Theory and Practice. Cambridge University Press

Janssen, A. , Pauls, T. (2005) A Monte Carlo comparison of studentized bootstrap and permutation tests for heteroscedastic two-sample problems. Computational Statistics 20, 369-383

Kijima, M. (1997) Markov processes for stochastic modeling. Chapman \& Hall

Lebart, L. (1969) Analyse statistique de la contiguïté. Publication de l'Institut de Statistiques de l'Université de Paris 18, 81-112

Leenders, R.T.A.J. (2002) Modeling social influence through network autocorrelation: Constructing the weight matrix. Social Networks 24, 21-47

LeSage, J.P., Pace, R.K. (2009) Introduction to spatial econometrics. Chapman \& Hall von Luxburg, U. (2007) A tutorial on spectral clustering. Statistics and Computing 17, $395-416$

Moran, P.A.P. (1950) Notes on continuous stochastic phenomena. Biometrika 37, 17-23

Sen, A., Smith, T. (1995) Gravity Models of Spatial Interaction Behavior. Springer

Thioulouse, J., Chessel, D. Champely, S. (1995) Multivariate analysis of spatial patterns: a unified approach to local and global structures. Environmental and Ecological Statistics, 2, 1-14

Tiefelsdorf, M., Boots, B. (1995) The exact distribution of Moran's I. Environment and Planning A 27, 985-999

Tiefelsdorf, M., Griffith, D. A. (2007) Semiparametric Filtering of Spatial Autocorrelation: The Eigenvector Approach. Environment and Planning A 39, 1193-221

Upton, G., Fingleton, B. (1985) Spatial Data Analysis by Example. Wiley

Waldhör, T. (1996) The spatial autocorrelation coefficient Moran's I under heteroscedasticity. Statistics in Medicine 15, 887-892 


\section{Appendix}

Proof of (7): $U$ being orthogonal, $\sum_{i} f_{i} c_{i \alpha} c_{i \beta}=\sum_{i} u_{i \alpha} u_{i \beta}=\delta_{\alpha \beta}$ and $\sum_{i} f_{i} c_{i \alpha}=$ $\sum_{i} \sqrt{f_{i}} u_{i \alpha}=\sum_{i} u_{i 0} u_{i \alpha}=\delta_{\alpha 0}$.

Proof of (9): independence implies the functional form $\sigma_{i j}=\delta_{i j} g\left(f_{i}\right)$ where $g(f)$ expresses a possible size dependence. Consider the aggregation of regions $j$ into super-region $J$, with aggregated field $X_{J}=\sum_{j \in J} f_{j} X_{j} / f_{J}$, where $f_{J}:=$ $\sum_{j \in J} f_{j}$. By construction,

$$
g\left(f_{J}\right)=\operatorname{Var}\left(X_{J}\right)=\frac{1}{f_{J}^{2}} \sum_{i, j \in J} f_{i} f_{j} \sigma_{i j}=\frac{1}{f_{J}^{2}} \sum_{j \in J} f_{j}^{2} g\left(f_{j}\right)
$$

that is $f_{J}^{2} g\left(f_{J}\right)=\sum_{j \in J} f_{j}^{2} g\left(f_{j}\right)$, with unique solution $g\left(f_{j}\right)=\sigma^{2} / f_{j}$ (and $\left.g\left(f_{J}\right)=\sigma^{2} / f_{J}\right)$, where $\sigma^{2}=\operatorname{Var}(\bar{X})$.

Proof of (10): $\hat{\sigma}_{\alpha \beta}:=\operatorname{Cov}\left(\hat{X}_{\alpha}, \hat{X}_{\beta}\right)=\sum_{i j} f_{i} f_{j} c_{i \alpha} c_{j \beta} \operatorname{Cov}\left(X_{i}, X_{j}\right)=$ $=\sigma^{2} \sum_{i} f_{i} c_{i \alpha} c_{i \beta}=\sigma^{2} \sum_{i} u_{i \alpha} u_{i \beta}=\sigma^{2} \delta_{\alpha \beta}$.

Proof of (11): $\sum_{\alpha \geq 1} \hat{x}_{\alpha}^{2}=\sum_{i j} \sqrt{f_{i} f_{j}} x_{i} x_{j} \sum_{\alpha \geq 0} u_{i \alpha} u_{j \alpha}-\hat{x}_{0}^{2}=\sum_{i} f_{i} x_{i}^{2}-\bar{x}^{2}=$ $\operatorname{var}(x)$. Also, $\operatorname{var}_{1 \mathrm{loc}}(x)=\frac{1}{2} \sum_{i j} e_{i j}\left(x_{i}-x_{j}\right)^{2}=\sum_{i} f_{i} x_{i}^{2}-\sum_{i j} e_{i j} x_{i} x_{j}=\sum_{i} f_{i} x_{i}^{2}-$ $\bar{x}^{2}-\sum_{\alpha \geq 1} \lambda_{\alpha} \sum_{i} c_{i \alpha} x_{i} \sum_{j} c_{j \alpha} x_{j}=\operatorname{var}(x)-\sum_{\alpha \geq 1} \lambda_{\alpha} \hat{x}_{\alpha}^{2}$.

Proof of (12) and (13): define

$$
a_{\alpha}:=\frac{\hat{x}_{\alpha}^{2}}{\sum_{\beta \geq 1} \hat{x}_{\beta}^{2}} \quad \text { with } \quad \sum_{\alpha \geq 1} a_{\alpha}=1 \quad \text { and } \quad I(\hat{x})=\sum_{\alpha \geq 1} \lambda_{\alpha} a_{\alpha} .
$$

Under $H_{0}$, the distribution of the non-trivial modes is exchangeable, i.e. $f(a)=$ $f(\pi(a))$. By symmetry, $E_{\pi}\left(a_{\alpha}\right)=1 /(n-1), E_{\pi}\left(a_{\alpha}^{2}\right)=s(x) /(n-1)^{2}$ where $s(x)=\sum_{\beta \geq 1} a_{\beta}^{2} /(n-1)$ and $E_{\pi}\left(a_{\alpha} a_{\beta}\right)=(1-s(x) /(n-1)) /[(n-1)(n-2)]$ for $\alpha \neq \beta$. Further substitution proves the result.

Proof of the semi-negative definitness of $Q$ in (20): for any vector $h$,

$$
0 \leq \frac{1}{2} \sum_{i j} \varepsilon_{i j}\left(h_{i}-h_{j}\right)^{2}=\sum_{i} \sigma_{i} h_{i}^{2}-\sum_{i j} \epsilon_{i j} h_{i} h_{j}=-\sum_{i j}\left(\epsilon_{i j}-\delta_{i j} \sigma_{j}\right) h_{i} h_{j} .
$$

Relation between the eigen-decompositions of $E^{s}(t)$ and $Q$ in (20): in matrix notation, $Q=\Pi^{\frac{1}{2}} R \Pi^{-\frac{1}{2}}$, and hence $Q \sqrt{f}=0$ by (19), showing $u_{0}=\sqrt{f}$ with $\mu_{0}=0$. Consider another, non-trivial eigenvector $u_{\alpha}$ of $Q$, with eigenvalue $\mu_{\alpha}$, orthogonal to $\sqrt{f}$ by construction. Identity $E(t)=\Pi \exp (t R)$ together with (5) yield

$$
E^{s}(t)=\sum_{k \geq 0} \frac{t^{k}}{k !} Q^{k}-\sqrt{f} \sqrt{f}^{\prime} \quad E^{s}(t) u_{\alpha}=\sum_{k \geq 0} \frac{t^{k} \mu_{\alpha}^{k}}{k !} u_{\alpha}=\exp \left(\mu_{\alpha} t\right) u_{\alpha}
$$

